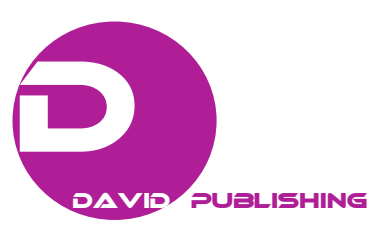

\title{
A New Approach to Electromagnetic Theories of Consciousness
}

\author{
Aibassov Erkin Zhakenovich ${ }^{1}$, Yemelyanova Valentina ${ }^{1}$, Savizky Ruben ${ }^{2}$ and Spataru Tudor ${ }^{2}$ \\ 1. Research Institute of New Chemical Technologies and Materials, Kazakh National University Al-Farabi, Almaty 005012, \\ Kazakhstan \\ 2. Columbia University, 3000 Broadway, New York, NY, 10027, USA
}

\begin{abstract}
The authors have proposed a new approach to the theory of consciousness, which takes into account the effect of the magnetic field on the process. The authors have shown that the process of information and thinking can be modeled on the basis of chemical thermodynamics. The authors offer general equations to calculate the thinking of the work of judgment the $L$ and of entropy solutions $G$ in the presence of a magnetic field. As a result, studies have shown that the magnetic effects strongly influence the thermodynamics of the process of thinking.
\end{abstract}

Key words: Magnetic effect, thermodynamics, process of thinking, consciousness.

\section{Introduction}

The electromagnetic theories of consciousness propose that consciousness can be understood as an electromagnetic phenomenon. Electromagnetic field theories of consciousness propose that consciousness results when a brain produces an electromagnetic field with specific characteristics. Susan Pockett and Johnjoe McFadden have proposed EM field theories [1-5].

The authors have previously been shown that the thermodynamic functions of internal energy $d U$ and free energy $d F$ in the presence of a magnetic field [5]:

$$
\begin{gathered}
d U=T d S+1 / 4 \pi H d B \\
d F=-S d T+1 / 4 \pi H d B
\end{gathered}
$$

It was also shown that the Nernst equation in a magnetic field changes, and is described by the equation [5]:

$$
E=E^{0}+R T / n F \ln a_{O x} / a_{\text {Red }}+1 / 4 \pi H d B
$$

The purpose of this work is an attempt to study the magnetic effects in thermodynamics thought processes and to find patterns of thinking mechanism in living organisms.

Corresponding author: Aibassov Erkin Zhakenovich, professor, research field: metal organic chemistry of uranium and thorium, $\mathrm{As}, \mathrm{Sb}$ and $\mathrm{Bi}$.

\section{Theory}

The starting point for McFadden and Pockett's theory is the fact that every time a neuron fires to generate an action potential, and a postsynaptic potential in the next neuron down the line, it also generates a disturbance in the surrounding electromagnetic field. McFadden views the brain's electromagnetic field as arising from the induced EM field of neurons. The synchronous firing of neurons is, in this theory, argued to amplify the influence of the brain's EM field fluctuations to a much greater extent than would be possible with the unsynchronized firing of neurons.

McFadden thinks that the EM field could influence the brain in a number of ways. Redistribution of ions could modulate neuronal activity, given that voltage-gated ion channels are a key element in the progress of axon spikes. Neuronal firing is argued to be sensitive to the variation of as little as one millivolt across the cell membrane, or the involvement of a single extra ion channel. Transcranial magnetic stimulation is similarly argued to have demonstrated that weak EM fields can influence brain activity.

Susan Pockett has advanced a theory, consciousness seen as identical to certain spatiotemporal patterns of 
the EM field. However, whereas McFadden argues that his deterministic interpretation of the EM field is not out-of-line with mainstream thinking, Pockett suggests that the EM field comprises a universal consciousness that experiences the sensations, perceptions, thoughts and emotions of every conscious being in the universe.

Thinking - certainly a biological phenomenon, and should therefore be subject to the atomic and molecular description. Science has established that the complex DNA and RNA molecules are capable of performing the function of storage and transmission of information is even more faith in the fact that the complex information processes, including thinking, made atomic-molecular mechanisms.

The fundamental thermodynamic properties of Shannon-type thinking is that the solution to the problem of information is not a process of spontaneous and necessary, i.e. running with decreasing free energy, but, on the contrary, requires the expenditure of work. This expresses the basic property information, that it is not deducible from the known data as a deduction - otherwise it would not be at all necessary, as always could be obtained from these data-but gives the new independent information that do not have believed to be reliable.

The process of logical thinking is similar to spontaneous thermodynamic process: In both cases, the original particle system (information) to be converted to a finite system of new particles or inferences. As spontaneous process in the thermodynamics with decreasing capacity of the free energy, and always leads to a more stable state. Spontaneous logical process flows down the free energy and gives the thermodynamically stable as a result of the withdrawal or deduction. The process of thinking is described thermodynamically expression:

Work information:

$$
I_{\text {Inform }}<0, \Delta \varphi_{\text {Inform }}<0
$$

The work of judgment (solution):

$$
L_{\text {Solution }}>0, \Delta \varphi_{\text {Solution }}>0
$$

where $\Delta \varphi$ - the fall of the free energy in the act of information or diskursii.

Between these extremes there is an intermediate area in which the magnitude of the fall of the free energy is large enough to process the judgment was spontaneous, but not high enough for it to be unique. This is an ambiguous area of probabilistic thinking.

In connection with the (Eq. (4)) increase in free energy of the information and hence its instability, it has to be stored in anti-entropy devices in memory, in the records of a particular type, including a variety of codes otherwise it will inevitably dissipate.

\section{Results and Discussion}

Dynamic energy $E=Z \lambda-\ni$ energy action in a certain space, energy vektorization energy; $\Psi$ - energy of the order.

Dynamic energy is manifested in two forms: vector $\mathrm{E}_{\mathrm{V}}=\mathrm{E \eta}$, giving direction and displacement of an object in its area of action and Brownian $E_{B}=E(1-\eta)$, extending the scope and status of the process.

Hence the total energy $U_{\text {Total }}=E_{V}+E_{B}+\Psi$

The information in contrast to the thinking can not appear as a product of pure inferences from other data. It is impossible, without spending any work, just standing on the platform, by "pure" reasoning to know address of your friend.

Information data are logically independent of each other, it is as if from nowhere output set of "primary", independent data (in physical chemistry - the system of non-interacting particles).

Thinking operates on the information data according to the laws of logic, and it resembles a chemical reaction "dissimilar" of the particles of the gas mixture to react with strictly defined laws.

The result of thinking is the conclusion that can be recorded. They include various elements of the original information.

Thinking there where begins the act of judgment as a result of a conscious selection of the source data come 
parcels in the form of some data (information), self-evident position (axioms) and certain assumptions (hypotheses), and subjecting them to an algorithm constructed in accordance with the laws of logic.

With this information system, axioms and hypotheses thinking process always leads to a definite conclusion. This is a very important property of thinking. It is similar to some spontaneous process, the outcome of which, regardless of the physical and chemical properties of the medium, where it occurs, is always the same!

It's like a roller coaster movement of rail road. Point of arrival is not dependent on anything. It is defined only by the desire to move the system to a stable state.

Thus, the thinking process is modeled on the basis of chemical thermodynamics in the form of spontaneous transition (Z-1) varieties of "chances" that are concentrated in a single cell in a k-th some sort of a drop in the free energy and entropy, expressed by the equation of thinking:

The work of judgment (Solution):

$$
L=\Sigma p_{i} \log p_{i}+\Delta \varphi^{o}=I_{V i n}+\Delta \varphi^{o}>0
$$

Entropy judgments (Solutions):

$$
G=\Sigma p_{i} \log p_{i}+\Delta H^{o}=H_{\text {Sannon }}+\Delta H^{o}>0
$$

The authors offer general equations to calculate the thinking of the work of judgment $L$ and entropy solution $G$ in the presence of a magnetic field:

$$
\begin{gathered}
L=I_{V i n}+\Delta \varphi+1 / 4 \pi H d B \\
G=H_{\text {Sannon }}+\Delta H+1 / 4 \pi H d B
\end{gathered}
$$

The final result of the act of thinking - a conclusion or inference - and there is a stable state of mind in the thermodynamic sense of the word.

Amazing uniqueness of the final result of mental activity proves it strictly directed, "spontaneous", the vector character, apparently independent from the chaos of Brownian motion of atoms and molecules that make up the substance of the brain or any other mechanism where there is thinking.

\section{Conclusions}

The analysis shows that the thinking process is modeled on the basis of chemical thermodynamics in the form of spontaneous transition (Z-1) varieties of "chances" that are concentrated in a single cell in a k-th some sort of a drop in the free energy and entropy, expressed by the equation of thought.

The authors offer general equations to calculate the thinking of the work of judgment $L$ and entropy solution $G$ in the presence of a magnetic field:

$$
\begin{aligned}
& L=I_{\text {Vin }}+\Delta \varphi+1 / 4 \pi H d B \\
& G=H_{\text {Sannon }}+\Delta H+1 / 4 \pi H d B
\end{aligned}
$$

The authors have shown that the process of thinking can be modeled on the basis of chemical thermodynamics. The authors offer general equations to calculate the thinking of the work of judgment the Land of entropy solutions $G$ in the presence of a magnetic field. As a result, studies have shown that the magnetic effects strongly influence the thermodynamics of the process of thinking. The consequence of the above is the ability to save the information entropy and speed of thinking in three dimensions, so the speed of thought to increase by several orders of magnitude.

\section{References}

[1] Johnjoe, M. F. 2002. "The Conscious Electromagnetic Information (Cemi) Field Theory: The Hard Problem Made Easy?" Journal of Consciousness Studies 9 (8): 45-60.

[2] Johnjoe, M. F. 2002. "Synchronous Firing and Its Influence on the Brain's Electromagnetic Field: Evidence for an Electromagnetic Field Theory of Consciousness." Journal of Consciousness Studies 9 (4): 23-50.

[3] Susan, P. 2000. The Nature of Consciousness, New York.

[4] William, R. U. 2014. Neural Theories of Mind: Why the Mind-Brain Problem May Never Be Solved.

[5] Mari, J., and Kunio, Y. 1995. Quantum Brain Dynamics and Consciousness. Notre Dame Seishin University, Okayama. 